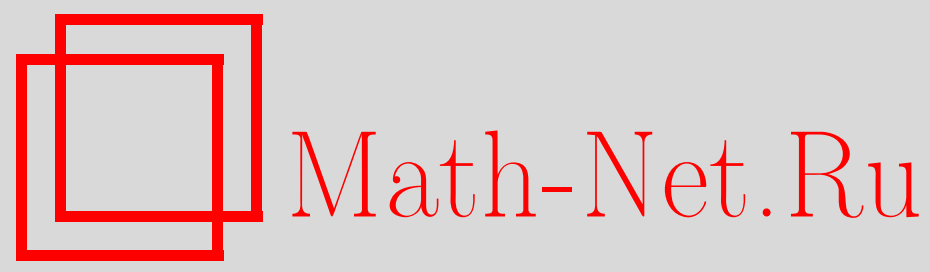

H.

K.

Мамадалиев,

Задача Трикоми для сильно-вырождающегося уравнения параболо-гиперболического типа, Матем. заметки, 1999, том 66, выпуск 3, 385-392

DOI: https://doi.org/10.4213/mzm1179

Использование Общероссийского математического портала Math-Net.Ru подразумевает, что вы прочитали и согласны с пользовательским соглашением http://www.mathnet.ru/rus/agreement

Параметры загрузки:

IP : 54.210 .77 .194

26 апреля 2023 г., 09:17:12

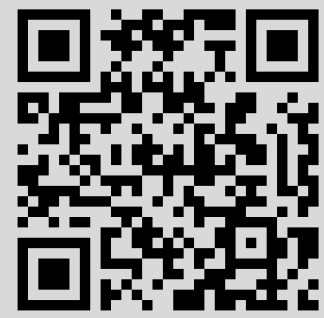


УДК 517.956.6

\section{ЗАДАЧА ТРИКОМИ ДЛЯ СИЛЬНО-ВЫРОЖДАЮЩЕГОСЯ УРАВНЕНИЯ ПАРАБОЛО-ГИПЕРБОЛИЧЕСКОГО ТИПА}

\section{Н. К. Мамадалиев}

Построено новое интегральное представление решения задачи Трикоми для сильно вырождающейся системы уравнений параболо-гиперболического типа.

Библиография: 5 названий.

Одной из основньх задач для уравнения смешанного типа является задача Трикоми, которая исследована многими авторами. Результаты исследований краевых задач для уравнений эллиптико-гиперболического типа второго рода в области, часть гранишы которой является линией вырождения, содержатся в работах [1]-[3].

В этой статье для уравнения гиперболического типа второго рода устанавливается представление обобщенного решения класса $R$ [3] и с помошью этого доказьвается однозначная разрешимость задачи Трикоми для уравнения параболо-гиперболического типа.

Рассмотрим уравнение

$$
\begin{aligned}
0=\left\{\begin{array}{l}
\frac{\partial^{2} \mathcal{U}}{\partial x^{2}}-\frac{\partial \mathcal{U}}{\partial y}+a(x, y) \mathcal{U}, \quad y \geqslant 0 \\
\frac{\partial^{2} \mathcal{U}}{\partial x^{2}}+y \frac{\partial^{2} \mathcal{U}}{\partial y^{2}}+\alpha \frac{\partial \mathcal{U}}{\partial y}, \quad y<0
\end{array}\right. \\
\alpha \in(-3,5 ;-3) \cup(-3 ;-2,5),
\end{aligned}
$$

в области $\mathcal{D}=\mathcal{D}_{1} \cup \mathcal{D}_{2} \cup A B$, причем область $\mathcal{D}_{1}$ ограничена при $y>0$ отрезками $A B$, $B B_{0}, A_{0} B_{0}, A A_{0}$ прямых $y=0, x=1, y=1, x=0$ соответственно, а область $\mathcal{D}_{2}$ при $y<0$ характеристиками уравнения (1):

$$
\begin{aligned}
& A C: \quad x-2 \sqrt{-y}=0, \quad B C: \quad x+2 \sqrt{-y}=1 \\
& A B: \quad y=0, \quad 0 \leqslant x \leqslant 1 .
\end{aligned}
$$

В уравнении (1) $a(x, y)$ - заданная функция, причем

$$
a(x, y) \in C^{(0, h)}\left(\mathcal{D}_{1}\right), \quad 0<h<1 .
$$


Задача Трикоми. Требуется определить в области $\mathcal{D}$ функцию $\mathcal{U}(x, y)$ со следующими свойствами:

а) $\mathcal{U}(x, y) \in C(\mathcal{D})$;

б) $\mathcal{U}(x, y)$ является регулярным решением уравнения $(1)$ в области $\mathcal{D}_{1}$ и обобщенным решением класса $R$ в области $\mathcal{D}_{2}$;

в) на линии вырождения выполняется условие склеивания

$$
\lim _{y \rightarrow-0}(-y)^{\alpha}\left[\mathcal{U}-A_{i}(\tau)\right]_{y}^{\prime}= \pm \lim _{y \rightarrow+0} \frac{\partial \mathcal{U}}{\partial y}
$$

при $i=3 \alpha \in(-3 ;-2,5)$, при $i=4 \alpha \in(-3,5 ;-3)$;

г) $\mathcal{U}(x, y)$ удовлетворяет граничным условиям

$$
\begin{gathered}
\left.\mathcal{U}\right|_{A A_{0}}=\varphi_{0}(y),\left.\quad \mathcal{U}\right|_{B B_{0}}=\varphi_{1}(y), \quad 0 \leqslant y \leqslant 1 \\
\left.\mathcal{U}\right|_{A C}=\psi(x)
\end{gathered}
$$

Здесь $A_{i}(\tau)$ - известный оператор, который указан ниже в $(2)$ и $(3), \varphi_{0}(y), \varphi_{1}(y)$ - заданные непрерывные функции, $0 \leqslant y \leqslant 1$, а $\psi(x)$ - заданная непрерьвная функция, причем $\psi^{(5)}(x)$ непрерьвна и $\psi(0)=\psi^{\prime}(0)=\psi^{\prime \prime}(0)=\psi^{\prime \prime \prime}(0)=\psi^{(\mathrm{IV})}(0)=0$.

ЗАмечание 1 . Рассмотрим уравнение $(1)$ в области $\mathcal{D}_{2}$, т.е. $L_{2} \mathcal{U}=0$. Непрерывное решение видоизмененной задачи Коши для уравнения $L_{2} \mathcal{U}=0$ в области $\mathcal{D}_{2}$ для значений $\alpha \in(-3 ;-2,5)$ с начальньми данными

$$
\begin{gathered}
\mathcal{U}(x, 0)=\tau(x) \\
\lim _{y \rightarrow-0}(-y)^{\alpha}\left[\mathcal{U}-A_{3}(\tau)\right]_{y}^{\prime}=\nu(x)
\end{gathered}
$$

в характеристических переменных $(\xi, \eta)$ имеет вид [1], [2]

$$
\begin{aligned}
& \mathcal{U}(\xi, \eta)= \gamma_{1}(\eta \xi)^{-2 p-5}\left[\int_{\xi}^{\eta} \tau(t)(\eta-t)^{p+2}(t-\xi)^{p+2} d t\right. \\
&+\frac{3}{2(p+3)(2 p+1)} \int_{\xi}^{\eta} \tau^{(2)}(t)(\eta-t)^{p+3}(t-\xi)^{p+3} d t \\
&+\frac{3}{4(p+3)(p+4)(2 p+1)(2 p+3)} \int_{\xi}^{\eta} \tau^{(4)}(t)(\eta-t)^{p+4}(t-\xi)^{p+4} d t \\
&+\frac{1}{8(p+3)(p+4)(p+5)(2 p+1)(2 p+3)(2 p+5)} \\
&\left.\times \int_{\xi}^{\eta} \tau^{(6)}(t)(\eta-t)^{p+5}(t-\xi)^{p+5} d t\right] \\
&+2^{2(2 p-1)} \gamma_{2} \int_{\xi}^{\eta} \nu(t)(\eta-t)^{-p}(t-\xi)^{-p} d t \\
& \equiv A_{3}(\tau)+2^{2(2 p-1)} \gamma_{2} \int_{\xi}^{\eta} \nu(t)(\eta-t)^{-p}(t-\xi)^{-p} d t, \\
& \tau(x) \in C^{(8)}[0,1], \quad \nu(x) \in C^{(2)}[0,1], \quad \xi=x-2 \sqrt{-y}, \quad \eta=x+2 \sqrt{-y}
\end{aligned}
$$




$$
\gamma_{2}=\frac{\Gamma(2 p+6)}{\Gamma^{2}(p+3)}, \quad \gamma_{2}=\left(\frac{1}{2}-p\right)^{-1} \frac{\Gamma(2-2 p)}{\Gamma^{2}(1-p)}, \quad p=\alpha-\frac{1}{2}
$$

Непрерьвное решение видоизмененной задачи Коши для уравнения $L_{2} \mathcal{U}=0$ в области $\mathcal{D}_{2}$ для значений $\alpha \in(-3,5 ;-3)$ с начальными данными

$$
\begin{gathered}
\mathcal{U}(x, 0)=\tau(x) \\
\lim _{y \rightarrow-0}(-y)^{\alpha}\left[\mathcal{U}-A_{4}(\tau)\right]_{y}^{\prime}=\nu(x)
\end{gathered}
$$

в характеристических переменных $(\xi, \eta)$ имеет вид [1], [2]

$$
\begin{aligned}
\mathcal{U}(\xi, \eta)= & \gamma_{1}(\eta-\xi)^{-2 p-7}\left(\int_{\xi}^{\eta} \tau(t)(\eta-t)^{p+3}(t-\xi)^{p+3} d t\right. \\
& +\frac{2}{(4+p)(2 p+1)} \int_{\xi}^{\eta} \tau^{(2)}(t)(\eta-t)^{p+4}(t-\xi)^{p+4} d t \\
& +\frac{3}{2(p+4)(p+5)(2 p+1)(2 p+3)} \int_{\xi}^{\eta} \tau^{(4)}(t)(\eta-t)^{p+5}(t-\xi)^{p+5} d t \\
& +\frac{1}{2(p+4)(p+5)(p+6)(2 p+1)(2 p+3)(2 p+5)} \\
& \times \int_{\xi}^{\eta} \tau^{(6)}(t)(\eta-t)^{p+6}(t-\xi)^{p+6} d t \\
& +\frac{1}{16(p+4)(p+5)(p+6)(p+7)(2 p+1)(2 p+3)(2 p+5)(2 p+7)} \\
& \left.\times \int_{\xi}^{\eta} \tau^{(8)}(t)(\eta-t)^{p+7}(t-\xi)^{p+7} d t\right) \\
& -2^{2(2 p-1)} \gamma_{2} \int_{\xi}^{\eta} \nu(t)(\eta-t)^{-p}(t-\xi)^{-p} d t \\
\equiv & A_{4}(\tau)-2^{2(2 p-1)} \gamma_{2} \int_{\xi}^{\eta} \nu(t)(\eta-t)^{-p}(t-\xi)^{-p} d t \\
\gamma_{1} & =\frac{\Gamma(2 p+8)}{\Gamma^{2}(p+4)}, \quad \gamma_{2}=\left(\frac{1}{2}-p\right)^{-1} \frac{\Gamma(2-2 p)}{\Gamma^{2}(1-p)}, \quad p=\alpha-\frac{1}{2} . \\
\nu(x) \in C^{(10)}[0,1], \quad C^{(2)}[0,1], \quad \xi=x-2 \sqrt{-y} & \eta \sqrt{-y}
\end{aligned}
$$

ОПрЕДЕЛЕниЕ. Функция $\mathcal{U}(\xi, \eta)$, определенная формулой $(2)$ или $(3)$, назьвается обобщенным решением уравнения $L_{2} \mathcal{U}=0$ класса $R$ в области $\mathcal{D}_{2}$, если $\nu(x) \in C[0,1]$ и функция $\tau(x)$ представима в виде

$$
\tau(x)=\int_{0}^{x}(x-t)^{-2 p} T(t) d t
$$

причем $T(t)$ - некоторая непрерьвная на $[0,1]$ функция. 
ЗАмЕчаниЕ 2 . Обобщенное решение $\mathcal{U} \in R$ непрерьвно в $\mathcal{D}_{2}$, а производные $\mathcal{U}_{x}$ и $\mathcal{U}_{y}$ непрерьвны в $\mathcal{D}_{2}$, причем функция $\left[\mathcal{U}-A_{i}(\tau)\right]_{y}^{\prime}, i=3,4$, непрерывна вплоть до линии изменения типа.

Справедливость замечания 2 нетрудно заметить на основании определения обобщенного решения класса $R$ и рассуждений работы [3]. На основании (4) находим

$$
\begin{aligned}
\tau^{(2)}(x)= & 2 p(2 p+1) \int_{0}^{x}(x-t)^{-2 p-2} T(t) d t, \\
\tau^{(4)}(x)= & 2 p(2 p+1)(2 p+2)(2 p+3) \int_{0}^{x}(x-t)^{-2 p-4} T(t) d t, \\
\tau^{(6)}(x)= & 2 p(2 p+1)(2 p+2)(2 p+3)(2 p+4)(2 p+5) \int_{0}^{x}(x-t)^{-2 p-6} T(t) d t, \\
\tau^{(8)}(x)= & 2 p(2 p+1)(2 p+2)(2 p+3)(2 p+4)(2 p+5)(2 p+6)(2 p+7) \\
& \times \int_{0}^{x}(x-t)^{-2 p-8} T(t) d t .
\end{aligned}
$$

ЗАмЕчАниЕ 3. Доказательство представления обобщенного решения класса $R$ при $\alpha \in(-3 ;-2,5)$ аналогично изменению параметра $\alpha \in(-2,5 ;-2)[4]$.

В этой работе получим представление обобщенного решения класса $R$ при $\alpha \in$ $(-3,5 ;-3)$. Подставляя полученные выше равенства и $(4)$ в $(3)$, имеем

$$
\begin{aligned}
\mathcal{U}(\xi, \eta)= & \gamma_{1}(\eta-\xi)^{-2 p-7}\left(\int _ { \xi } ^ { \eta } \left(\int _ { 0 } ^ { t } T ( \zeta ) \left((\eta-t)^{p+3}(t-\xi)^{p+3}(t-\zeta)^{-2 p}\right.\right.\right. \\
& +\frac{4 p}{p+4}(\eta-t)^{p+4}(t-\xi)^{p+4}(t-\zeta)^{-2 p-2} \\
& +\frac{6 p(p+1)}{(p+4)(p+5)}(\eta-t)^{p+5}(t-\xi)^{p+5}(t-\zeta)^{-2 p-4} \\
& +\frac{4 p(p+1)(p+2)}{(p+4)(p+5)(p+6)}(\eta-t)^{p+6}(t-\xi)^{p+6}(t-\zeta)^{-2 p-6} \\
& \left.\left.\left.+\frac{p(p+1)(p+2)(p+3)}{(p+4)(p+5)(p+6)(p+7)}(\eta-t)^{p+7}(t-\xi)^{p+7}(t-\zeta)^{-2 p-8}\right) d \zeta\right) d t\right) \\
& -2^{2(2 p-1)} \gamma_{2} \int_{\xi}^{\eta} \nu(t)(\eta-t)^{-p}(t-\xi)^{-p} d t \\
\equiv & \gamma_{1}(\eta-\xi)^{-2 p-7 \mathfrak{I}_{1}-\mathfrak{I}_{2} .}
\end{aligned}
$$

В выражении для $\mathfrak{I}_{1}$ промежуток интегрирования по $\zeta$ разобьем на два, $(0, \xi)$ и $(\xi, t)$. Затем, меняя порядок интегрирования, получим

$$
\mathfrak{I}_{1}=\int_{0}^{\xi} I_{1}(\xi, \eta ; \zeta) T(\zeta) d \zeta+\int_{\xi}^{\eta} I_{2}(\xi, \eta ; \zeta) T(\zeta) d \zeta
$$

где

$$
I_{1}(\xi, \eta ; \zeta)=\int_{\xi}^{\eta}(\eta-t)^{p+3}(t-\xi)^{p+3}(t-\zeta)^{-2 p} d t
$$




$$
\begin{aligned}
& +\frac{4 p}{p+4} \int_{\xi}^{\eta}(\eta-t)^{p+4}(t-\xi)^{p+4}(t-\zeta)^{-2 p-2} d t \\
& +\frac{6 p(p+1)}{(p+4)(p+5)} \int_{\xi}^{\eta}(\eta-t)^{p+5}(t-\xi)^{p+5}(t-\zeta)^{-2 p-4} d t \\
& +\frac{4 p(p+1)(p+2)}{(p+4)(p+5)(p+6)} \int_{\xi}^{\eta}(\eta-t)^{p+6}(t-\xi)^{p+6}(t-\zeta)^{-2 p-6} d t \\
& +\frac{p(p+1)(p+2)(p+3)}{(p+4)(p+5)(p+6)(p+7)} \int_{\xi}^{\eta}(\eta-t)^{p+7}(t-\xi)^{p+7}(t-\zeta)^{-2 p-8} d t \\
I_{2}(\xi, \eta ; \zeta)= & \int_{\zeta}^{\eta}(\eta-t)^{p+3}(t-\zeta)^{-2 p}(t-\xi)^{p+3} d t \\
& +\frac{4 p}{p+4} \int_{\zeta}^{\eta}(\eta-t)^{p+4}(t-\zeta)^{-2 p-2}(t-\xi)^{p+4} d t \\
& +\frac{6 p(p+1)}{(p+4)(p+5)} \int_{\zeta}^{\eta}(\eta-t)^{p+5}(t-\zeta)^{-2 p-4}(t-\xi)^{p+5} d t \\
& +\frac{4 p(p+1)(p+2)}{(p+4)(p+5)(p+6)} \int_{\zeta}^{\eta}(\eta-t)^{p+6}(t-\zeta)^{-2 p-6}(t-\xi)^{p+6} d t \\
& +\frac{p(p+1)(p+2)(p+3)}{(p+4)(p+5)(p+6)(p+7)} \int_{\zeta}^{\eta}(\eta-t)^{p+7}(t-\zeta)^{-2 p-8}(t-\xi)^{p+7} d t .
\end{aligned}
$$

Для вычисления этих выражений используем интегральное представление гипергеометрических функций [3]:

$$
\begin{aligned}
& \int_{\xi}^{\eta}(\eta-t)^{k}(t-\xi)^{l}(t-\zeta)^{m} d t \\
& \quad=\frac{\Gamma(k+1) \Gamma(l+1)}{\Gamma(k+l+2)}(\eta-\xi)^{k+l+1}(\eta-\zeta)^{m} F\left(k+1,-m, k+l+2 ; \frac{\eta-\xi}{\eta-\zeta}\right) .
\end{aligned}
$$

Имеем

$$
\begin{aligned}
I_{1}= & \frac{\Gamma^{2}(p+4)}{\Gamma(2 p+8)}(\eta-\xi)^{2 p+7}(\eta-\zeta)^{-2 p}(F(p+4,2 p, 2 p+8 ; z) \\
& +\frac{2 p}{2 p+9} z^{2} F(p+5,2 p+2,2 p+10 ; z) \\
& +\frac{3 p(p+1)}{2(2 p+9)(2 p+11)} z^{4} F(p+6,2 p+4,2 p+12 ; z) \\
& +\frac{p(p+1)(p+2)}{2(2 p+9)(2 p+11)(2 p+13)} z^{6} F(p+7,2 p+6,2 p+14 ; z) \\
& \left.+\frac{p(p+1)(p+2)(p+3)}{16(2 p+9)(2 p+11)(2 p+13)(2 p+15)} z^{8} F(p+8,2 p+8,2 p+16 ; z)\right) \\
& z=\frac{\eta-\xi}{\eta-\zeta}
\end{aligned}
$$




$$
\begin{aligned}
I_{2}= & \frac{p(p+1)(p+2)(p+3) \Gamma(p+4) \Gamma(-2 p-7)}{\Gamma(1-p)}(\eta-\zeta)^{-p}(\eta-\xi)^{p+7} \\
& \times\left(F\left(p+8,-p-7,1-p ; z_{1}\right)+\frac{8(2 p+7)}{1-p} z_{1} F\left(p+7,-p-6,2-p ; z_{1}\right)\right. \\
& +\frac{24(2 p+7)(2 p+5)}{(1-p)(2-p)} z_{1}^{2} F\left(p+6,-p-5,3-p ; z_{1}\right) \\
& +\frac{32(2 p+7)(2 p+5)(2 p+3)}{(1-p)(2-p)(3-p)} z_{1}^{3} F\left(p+5,-p-4,4-p ; z_{1}\right) \\
& \left.+\frac{16(2 p+7)(2 p+5)(2 p+3)(2 p+1)}{(1-p)(2-p)(3-p)(4-p)} z_{1}^{4} F\left(p+4,-p-3,5-p ; z_{1}\right)\right), \\
& z_{1}=\frac{\eta-\zeta}{\eta-\xi} .
\end{aligned}
$$

Лемма. Справедливы тождества:

$$
\begin{aligned}
F(p+ & 4,2 p, 2 p+8 ; z)+\frac{2 p}{2 p+9} z^{2} F(p+5,2 p+2,2 p+10 ; z) \\
& +\frac{3 p(p+1)}{2(2 p+9)(2 p+11)} z^{4} F(p+6,2 p+4,2 p+12 ; z) \\
& +\frac{p(p+1)(p+2)}{2(2 p+9)(2 p+11)(2 p+13)} z^{6} F(p+7,2 p+6,2 p+14 ; z) \\
& +\frac{p(p+1)(p+2)(p+3)}{16(2 p+9)(2 p+11)(2 p+13)(2 p+15)} z^{8} F(p+8,2 p+8,2 p+16 ; z)=(1-z)^{-p}, \\
F(p+ & \left.8,-p-7,1-p ; z_{1}\right)+\frac{8(2 p+7)}{1-p} z_{1} F\left(p+7,-p-6,2-p ; z_{1}\right) \\
& +\frac{24(2 p+7)(2 p+5)}{(1-p)(2-p)} z_{1}^{2} F\left(p+6,-p-5,3-p ; z_{1}\right) \\
& +\frac{32(2 p+7)(2 p+5)(2 p+3)}{(1-p)(2-p)(3-p)} z_{1}^{3} F\left(p+5,-p-4,4-p ; z_{1}\right) \\
& +\frac{16(2 p+7)(2 p+5)(2 p+3)(2 p+1)}{(1-p)(2-p)(3-p)(4-p)} z_{1}^{4} F\left(p+4,-p-3,5-p ; z_{1}\right)=\left(1-z_{1}\right)^{-p} .
\end{aligned}
$$

ДоКАЗАТЕЛЬСТВо тождеств аналогично изложенному в работе [5].

На основании леммы выражения $I_{1}(\xi, \eta ; \zeta)$ и $I_{2}(\xi, \eta ; \zeta)$ запишем в виде

$$
\begin{aligned}
& I_{1}(\xi, \eta ; \zeta)=\frac{\Gamma^{2}(p+4)}{\Gamma(2 p+8)}(\eta-\xi)^{2 p+7}(\eta-\zeta)^{-p}(\xi-\zeta)^{-p} \\
& I_{2}(\xi, \eta ; \zeta)=\frac{p(p+1)(p+2)(p+3) \Gamma(p+4) \Gamma(-2 p-7)}{\Gamma(1-p)}(\eta-\xi)^{2 p+7}(\eta-\zeta)^{-p}(\zeta-\xi)^{-p}
\end{aligned}
$$

Подставив $(6),(7)$ в $(5)$, получим представление обобщенного решения класса $R$

$$
\mathcal{U}(\xi, \eta)=\int_{0}^{\xi}(\eta-\zeta)^{-p}(\xi-\zeta)^{-p} T(\zeta) d \zeta+\int_{\xi}^{\eta}(\eta-\zeta)^{-p}(\zeta-\xi)^{-p} N(\zeta) d \zeta
$$


где

$$
N(\zeta)=\frac{1}{2 \cos \pi p} T(\zeta)-2^{4 p-2} \gamma_{2} \nu(\zeta) .
$$

Для всех рассматриваемых значений $\alpha$ представление обобщенного решения класса $R$ получено впервые. Задача Трикоми для эллиптико-гиперболического уравнения при $\alpha<1$ и $\alpha=-n+\alpha_{0}, 1 / 2<\alpha_{0}<1, n=0,1,2, \ldots$, изучена в работе [2].

Используя условие на характеристике $A C$, согласно п. г) в постановке задачи Трикоми из обобщенного решения (8) имеем

$$
\int_{0}^{\eta}(\eta-\zeta)^{-p} \zeta^{-p} N(\zeta) d \zeta=\psi\left(\frac{\eta}{2}\right) .
$$

Применяя к обеим частям выражения $(10)$ оператор $\mathcal{D}_{0 \eta}^{1-p}$, получим

$$
N(\eta)=\zeta^{p} \frac{1}{\Gamma(1-p)} \mathcal{D}_{0 \eta}^{1-p} \psi\left(\frac{\eta}{2}\right) .
$$

Далее с учетом (9) и (10), подставляя значение $T(\zeta)$ в представление (4), получим основное соотношение между $\tau(x)$ и $\nu(x)$ на отрезке $A B$ при $y \rightarrow-0$

$$
\begin{aligned}
\tau(x)= & -2^{4 p-2} \gamma_{2} \cos \pi p \int_{0}^{x} \nu(t)(x-t)^{-2 p} d t \\
& +\frac{2 \cos \pi p}{\Gamma(1-p)} \int_{0}^{x}(x-t)^{-2 p} t^{p} \mathcal{D}_{0 t}^{1-p} \psi\left(\frac{t}{2}\right) d t .
\end{aligned}
$$

Для получения соотношения между функциями $\tau(x)$ и $\nu(x)$ на отрезке $A B$ при $y \rightarrow+0$ перейдем в уравнении (1) к пределу при $y \rightarrow+0$. Имеем

$$
\tau^{\prime \prime}(x)-\nu(x)+a(x, 0) \tau(x)=0 .
$$

Исключая из (11) и (12) функцию $\tau(x)$, получим интегральное уравнение относительно функции $\nu(x)$, эквивалентное задаче Трикоми:

$$
\begin{aligned}
\nu(x) \pm & 2 p(2 p+1) 2^{4 p-2} \gamma_{2} \cos \pi p \int_{0}^{x} \nu(t)(x-t)^{-2 p-2} d t \\
& \pm 2^{4 p-2} \gamma_{2} \cos \pi p a(x, 0) \int_{0}^{x} \nu(t)(x-t)^{-2 p} d t \\
= & \pm \frac{2 \cos \pi p}{\Gamma(1-p)} 2 p(2 p+1) \int_{0}^{x}(x-t)^{-2 p-2} t^{p} \mathcal{D}_{0 t}^{1-p} \psi\left(\frac{t}{2}\right) d t \\
& \pm \frac{2 \cos \pi p}{\Gamma(1-p)} a(x, 0) \int_{0}^{x}(x-t)^{-2 p} t^{p} \mathcal{D}_{0 t}^{1-p} \psi\left(\frac{t}{2}\right) d t, \quad 0 \leqslant x \leqslant 1 .
\end{aligned}
$$

В силу ограничений, наложенных на функцию $\psi(x)$, правая часть непрерьвна. Так как $6<-2 p<7$ при $\alpha \in(-3 ;-2,5)$ и $7<-2 p<8$ при $\alpha \in(-3,5 ;-3)$, ядро интегрального уравнения (13) также непрерьвно. Таким образом, (13) является интегральным уравнением Вольтерра второго рода относительно функции $\nu(x)$ с непрерьвным ядром и непрерьвной правой частью. Решение полученного уравнения существует и единственно [6]. Следовательно, задача Трикоми однозначно разрешима.

ЗАмЕчАниЕ 4. Полученные результаты справедливы и при выполнении следующих условий склеивания на линии вырождения:

$$
\lim _{y \rightarrow-0}(-y)^{\alpha}\left[\mathcal{U}-A_{i}(\tau)\right]_{y}^{\prime}=-\lim _{y \rightarrow+0} \frac{\partial \mathcal{U}}{\partial y} .
$$

Доказательство проводится аналогично. 


\section{СПИСОК ЦИТИРОВАННОЙ ЛИТЕРАТУРЫ}

[1] Терсенов С. А. Введение в теорию уравнений, вырождающихся на границе. Новосибирск: НГУ, 1973.

[2] Исамухамедов С. С. Некоторые краевые задачи для уравнений смешанного типа второго рода. Автореф. дисс. ... к.ф.-м.н. Ташкент, 1975.

[3] Смирнов М. М. Уравнения смешанного типа. М.: Наука, 1970.

[4] Мамадалиев Н. К., Нагорный А. М. Задача Трикоми для уравнения параболо-гиперболического типа с характеристической линией изменения типа // Тезисы докл. Всесоюзной конференции "Краевые задачи и их спектральные вопросы для дифференциальных уравнений". Алма-Ата, 1991.

[5] Мамадалиев Н. К. Аналог задачи Трикоми для сильно вырождающегося уравнения параболо-гиперболического типа // Докл. АН УзССР. 1991. № 4. С. 4-6.

[6] Трикоми Ф.О. Интегральные уравнения. М.: ИЛ, 1960.

Ташкентский государственный университет

Поступило

13.02 .96 